\title{
Rho GTPase Inactivation Impairs Lens Growth and Integrity
}

\author{
Vasantha Rao, Eric Wawrousek, Ernst R. Tamm, and Samuel Zigler, Jr. \\ Department of Ophthalmology (VR) and Department of Pharmacology and Cancer Biology (VR), Duke University \\ Medical School, Durham, North Carolina; National Eye Institute (EW, SZ), National Institutes of Health, Bethesda, \\ Maryland; and Anatomisches Institut (ERT), Lehrstuhl II, Universitaet Erlangen-Nuernberg, Germany
}

SUMMARY: To elucidate the significance of Rho GTPase signaling on lens growth and structural integrity, we have selectively inactivated Rho GTPase in the ocular lens. To achieve this tissue-specific inactivation, a transgene encoding the C3-exoenzyme from Clostridium botulinum has been expressed in mice under transcriptional control of the lens-specific $\alpha \mathrm{A}$-crystallin promoter. C3-exoenzyme is known to selectively inactivate all Rho GTPase isoforms by ADP-ribosylating an asparagine residue at position 41. Mice expressing the C3-exoenzyme transgene exhibited selective ocular defects, including cataract and microphthalmia. Extralenticular effects included ocular hemorrhage (blood accumulation in the anterior and posterior chambers of the eye) and abnormalities of the iris including focal attachments to lens and cornea (synechiae). C3-transgene expression was found only in the lens and not in the other ocular tissues as determined by RT-PCR analysis. Histologic examination of the eyes of C3 transgenic mice from two independent lines revealed extensive abnormalities of the lens, including defective fiber cell differentiation and elongation, ruptured posterior lens capsule, and thickened anterior lens capsule. Electron microscopic analysis of hemorrhaged C3 eyes showed abnormalities in the posterior hyaloid vessels. Collectively these data reveal the importance of Rho GTPase signaling in regulating lens growth and maintenance of lens transparency. (Lab Invest 2002, 82:231-239).

$T$ he normal development and growth of the vertebrate lens is a complex process that requires the precise spatial and temporal control of proliferation of epithelial cells and their differentiation into fiber cells. Fiber cell differentiation is accompanied by cell elongation and remodeling of cytoskeletal structures, cessation of DNA replication and of cell division, expression of lens fiber-specific proteins such as the $\beta$ and $\gamma$ crystallins, and finally the loss of nuclei and other subcellular organelles during the late stages of maturation (McAvoy, 1978; Piatigorsky, 1981). Although insights have emerged regarding genes controlling the early stages of lens formation, little is known about lens fiber cell differentiation and elongation. Lens epithelial cells maintain a lifelong commitment to the programs of proliferation and differentiation. Therefore knowledge of the inductive factors that regulate these processes, and more importantly, the signaling pathways that serve to transduce the biologic information conveyed by these external factors (such as growth factors and hormones) into appropriate responses in cells of the developing lens is critical for understanding both normal and deranged lens development.

Received December 10, 2001.

This work was supported by United States Public Health Service grant R01-EY12201 (VR) and SFB539 and IZKF (ERT).

Address reprint requests to: Dr. P. Vasantha Rao, Department of Ophthalmology, Duke University Medical Center, Erwin Road, P.O. Box 3802, Durham,NC27710-3802.E-mail:rao00011@mc.duke.edu
Members of the Rho family of GTPases (Rac, CDC42, and Rho) are known to regulate cell motility, morphology, adhesion, cytokinesis, and gene expression through their interactions with the cellular actin network (Alest and D'Souza-Schorey, 1997; Hall, 1998). The formation of filopodia and lamellipodia, for instance, involves CDC42 and Rac, respectively, whereas assembly of focal adhesions and stress fibers is regulated by Rho (Hall, 1998). Because the actin cytoskeleton constitutes a primary target of the Rho GTPases, the cytoskeleton could be intimately involved in signaling pathways regulating lens epithelial cell elongation and differentiation.

We hypothesize that Rho GTPases play an important role in the regulation of lens growth and development and in the maintenance of lens transparency. To address this possibility, we initially characterized expression and distribution profiles of the Ras-related GTP-binding proteins (Maddala et al 2001a; Rao et al, 1997b) and accessory proteins regulating Ras and Rho GTPase function, including Ras-GAP, Rho-GAP, and Rho GDI, in different regions of the lens. We also found that lovastatin, which prevents isoprenylation and thereby membrane localization and function of the small GTPases, causes extensive morphologic alterations and impairs cell proliferation in lens epithelial cell cultures and induces opacification in organ cultured lenses (Maddala et al, 2001b; Rao et al, 1997a). Based on these preliminary observations in the lens, together with the currently understood biologic roles of the Rho GTPases in general, our goal in this study was to elucidate the effects of Rho GTPase on lens growth, development, and integrity. To that end, we 
have produced transgenic mice in which the Rho GTPase has been specifically inactivated in the lens. This report describes the ocular phenotypes and histologic changes in the C3 transgenic mice.

\section{Results}

\section{Generation of C3 Transgenic Mice}

To explore the importance of Rho GTPase signaling in the regulation of lens development, growth, and function, we have generated a transgenic mouse model in which Rho GTPase function has been targeted by tissue-specific expression of C3-exoenzyme in the ocular lens. C3-exoenzyme from Clostridium botulinum specifically and irreversibly inactivates Rho GTPase ( $A, B$, and $C$ isoforms) through ADP-ribosylation (Dillon and Feig, 1995; Rubin et al, 1988; Yamamoto et al, 1993), generating ADP-ribosylated Rho GTPase, which can behave in a dominant negative manner by competing for downstream effector molecules (Yamamoto et al, 1993). The mouse $\alpha \mathrm{A}$-crystallin promoter was used to drive C3-exoenzyme expression in the lens. This promoter, the expression of which is confined mainly to lens fibers, has been used extensively to drive expression of various transgenes in the lens (Lovicu and Overbeek, 1998; Robinson et al, 1995; Stolen et al, 1997; Wawrousek et al, 1990). Three transgenic lines designated ODO4-11, 16, and 21 were established using the plasmid construct depicted in Figure 1.

\section{Expression and Functional Integrity of Transgene Protein Product}

Expression of C3-exoenzyme mRNA was confirmed by RT-PCR analysis of total lens RNA extracted from 3-week-old mice for line ODO4-11; and for line ODO421 , single lenses obtained from 4-day and 6-week-old

A

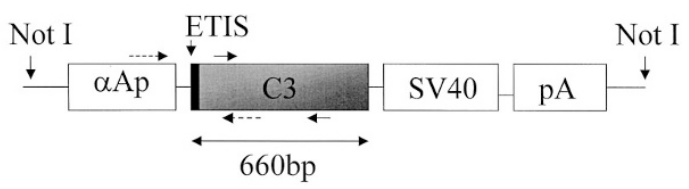

B

\section{$\stackrel{\text { CCACCATGGCTTATTCAAATACTTACCAGGAGTtTAC }}{\longrightarrow}$ ETIS C3 sequence}

Figure 1.

A, Structure of the $\alpha \mathrm{A} / \mathrm{C} 3$ transgene. Bacterial C3 cDNA (coding region) containing a eukaryotic translation initiation sequence was subcloned into the polylinker region of the CPV2 plasmid between the $\alpha$ A-crystallin promoter and the SV40 small $t$ antigen intron and polyadenylation sequences. The locations of primers used for screening genomic tail DNA and for RT-PCR analysis are shown in hatched and solid arrows, respectively. B, Sequence of the oligonucleotide representing the eukaryotic translation initiation site (large caps) inserted upstream of the C3 coding sequence (small caps). $\alpha \mathrm{Ap}=$ $\alpha \mathrm{A}$-crystallin promoter; ETIS $=$ Eukaryotic translation initiation sequence; $\mathrm{pA}$ $=$ Polyadenylation. mice were pooled (Fig. 2A). Although lenses obtained from line ODO4-11 and line ODO4-21 mice showed the expected 316-bp (partial fragment) PCR product amplified using C3-exoenzyme specific primers, line ODO4-16 mice showed no detectable C3 product. Reactions performed in the absence of reverse transcriptase (RT) showed no C3 product, indicating the amplified C3 product is not a result of DNA contamination; furthermore, before $\mathrm{RT}$ reaction, total RNA was also treated with DNase-1. Because C3 mice exhibit some extralenticular ocular phenotype, C3exoenzyme transgene expression in other ocular tissues was tested by first removing the lens and then performing RT-PCR analysis on the rest of the eye. No expression of C3-exoenzyme message was observed, although GAPDH expression was detected, indicating
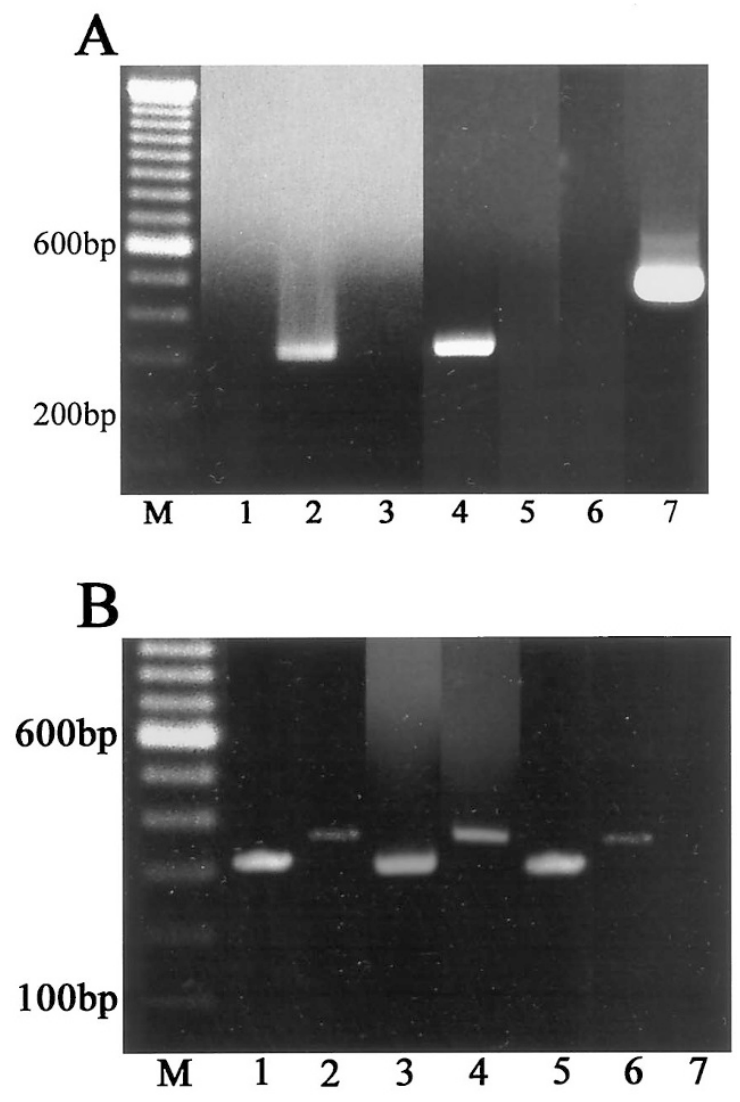

Figure 2.

Expression of C3 transgene product. A, Total RNA extracted from a single pair or from pooled lenses from wild-type littermate controls and from 0D04-11, OD04-16, and 0D04-21 transgene-positive mice was used for RT-PCR analysis using C3-specific primers. Lanes 1 to 7 represent the following: 1 , wild-type littermate lens; 2, OD04-11 mouse lens; 3, -RT control to OD04-11 lens sample; 4, 0D04-21 mouse lens; 5, 0D04-16 mouse lens; 6 , nonlenticular ocular tissues of line 0D04-11; and 7, GADPH expression in nonlenticular ocular tissues of line 0D04-11 mouse. The expected 316-bp fragment was observed in line 0D04-11 and 0D04-21 lenses (lanes 2 and 4, respectively) but not in line 0D04-16 (lane 5) or in wild-type littermate lenses (lane 1). C3 transgene expression was found to be lens specific and showed no expression in nonlenticular ocular tissues (lane 6). Lane $M$ indicates base pair marker. B, Expression of RhoA and RhoB in lenses of wild-type (lanes 1 and 2, respectively), transgenic line 0D04-11 (lanes 3 and 4), and line 0D04-21 mice (lanes 5 and 6). Lane 7 indicates - RT control. Lane $M$ is base pair marker. These data indicate that RhoA and RhoB expression were not affected by the C3 transgene in line 0D04-11 and 0D04-21 mice. 
positive RT reaction. Both C3 transgenic and wildtype lenses showed normal expression of RhoA and RhoB as confirmed by RT-PCR analysis (Fig. 2B).

To demonstrate the presence of the transgene protein product, lenses from neonatal mice (lines ODO4-11 and ODO4-21) were isolated and homogenized, and the homogenates were subjected to SDSPAGE analysis and Western blotting using a monoclonal antibody raised against C3-exoenzyme (Yamamoto et al, 1993) kindly provided by Shunji Kozaki, Department of Veterinary Science, University of Osaki Prefecture, Japan. The antibody recognized a single protein of $\approx 25 \mathrm{kd}$ in the transgenic samples (mostly in the insoluble fractions) but not in the normal littermate controls (Fig. 3A). In older lenses it migrated as a doublet of approximately $50 \mathrm{kd}$ (data not shown).

To confirm the functional integrity of the C3exoenzyme expressed in the transgenic lenses, in vitro ADP-ribosylation assays were performed using $\left[{ }^{32} \mathrm{P}\right] N A D$. A 26-kd ADP-ribosylated protein corresponding to Rho was detected in wild-type control samples and was markedly reduced in transgenic samples, indicating that most of the Rho GTPase protein was already endogenously ADP ribosylated by transgenically produced C3-exoenzyme (Fig. 3B).

\section{Ocular Phenotypes of C3 Transgenic Mice}

The ODO4-11 and ODO4-21 founder mice and their progeny exhibited severe ocular abnormalities including cataract, microphthalmia, and ocular hemorrhage evident as early as embryonic day 15 . These effects are shown in 6-week-old mice (ODO4-21) by slitlamp photography in Figure 4. The cataracts appear to be cortical in nature. There was variability in the severity of these effects, for example, microphthalmia was not present in all affected animals and was not always bilateral in line ODO4-21, whereas line ODO4-11 mice consistently show bilaterally smaller eyes. In many instances dilation of the pupils of the transgenic mice was very limited when compared with littermate wildtype mice (Fig. 5a, panels $\mathrm{C}$ and D). Compared with lines ODO4-11 and ODO4-21, the founder and prog- eny of line ODO4-16 revealed no obvious phenotype and eyes appeared normal. This is consistent with the failure to detect expression of C3 in RT-PCR analysis in ODO4-16 mice. Line ODO4-21 mice that had severe ocular phenotype (both males and females) including cataract and ocular hemorrhage, failed to breed after a couple of progeny and could not be maintained. On the other hand, all line ODO4-11 C3-positive mice were males, bilaterally microphthalmic, and cataractous with ocular hemorrhage. Notably, the percentage of male progeny in line ODO4-11 was consistently low (only around $20 \%$ ) as compared with wild-type mice (50\%). Although C3 transgenic mice have a normal growth rate and no other phenotype, the inability to breed line ODO4- 21 and the consistently lower percentage of males that are always C3 positive in line ODO4-11 suggest some systemic stress in these mice. Weights $(\mathrm{mg})$ of whole eye obtained from 6- to 10-month-old mice $(22.83 \pm 1.11, n=14$ wild type versus $12.35 \pm 0.448, n=13$, C3 mice) and lens weights obtained from 6 -week-old mice (5.08 \pm 0.405 wild type $n=8$ vs $2.76 \pm 0.44 n=7$, C3) were reduced by $50 \%$ in $\mathrm{C} 3$ mice (ODO4-11) as compared with littermate controls. For line ODO4-21 mice, there was not enough sample number available for these measurements.

\section{Histologic Changes in C3 Transgenic Mice}

To evaluate early changes in developing C3 lenses, histologic analysis was performed using eyes from embryonic, neonatal, and postnatal mice (Fig. 5, a and b). Embryonic lens sections (E15.5) exhibited abnormal primary fiber cell organization and shorter fiber lengths, with accumulation of vacuoles and gaps between the cells (Fig. 5a, panel E, and Fig. 5b, panel D); lens size was also much smaller than controls. Neonatal (4 day old) C3 lenses showed abnormal fiber cell organization and frequently a ruptured posterior capsule (Fig. 5a, panel F). Although the secondary fibers close to the equatorial region appeared to be quite normal, the organization of the primary fibers was very different from the littermate controls. Fiber
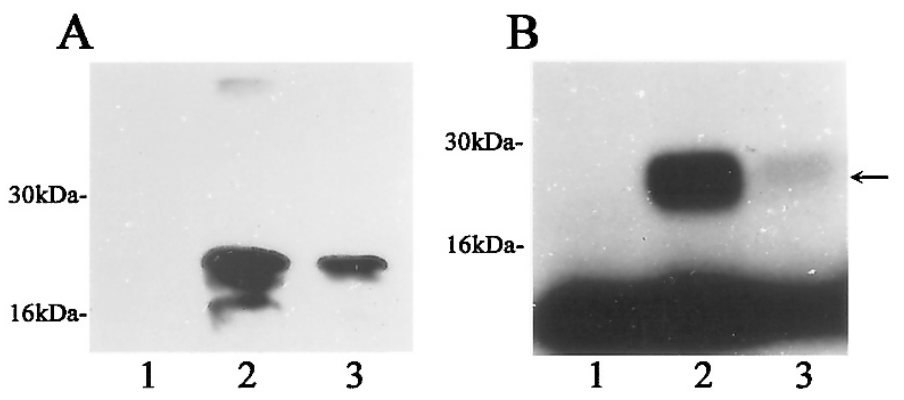

\section{ADP Ribosylated Rho}

Figure 3.

A, Detection of C3 protein by Western blot analysis. Although homogenates obtained from wild-type lens (lane 1) showed no immunoreactivity to C3 monoclonal antibody, lens homogenates obtained from line 0D04-11 (lane 2) and line 0D04-21 (lane 3) C3 mice showed a immunopositive band ( $\approx 25 \mathrm{kDa}$ ). B, To test the functional integrity of the C3 protein, lens homogenates from transgenic and wild-type littermates were subjected to in vitro ADP-ribosylation of Rho GTPase by exogenous C3-exoenzyme. Lane 1 is a control in which the C3-exoenzyme was absent. As compared with a wild-type sample (lane 2), transgenic lens homogenate obtained from line 0D04-21 mice showed a very weak in vitro ADP-ribosylated band (lane 3), indicating that most of the Rho GTPase had already been ribosylated by the endogenous C3 produced transgenically, suggesting its in vivo inactivation. The lower dark band is derived from the unbound radiolabeled compound. 

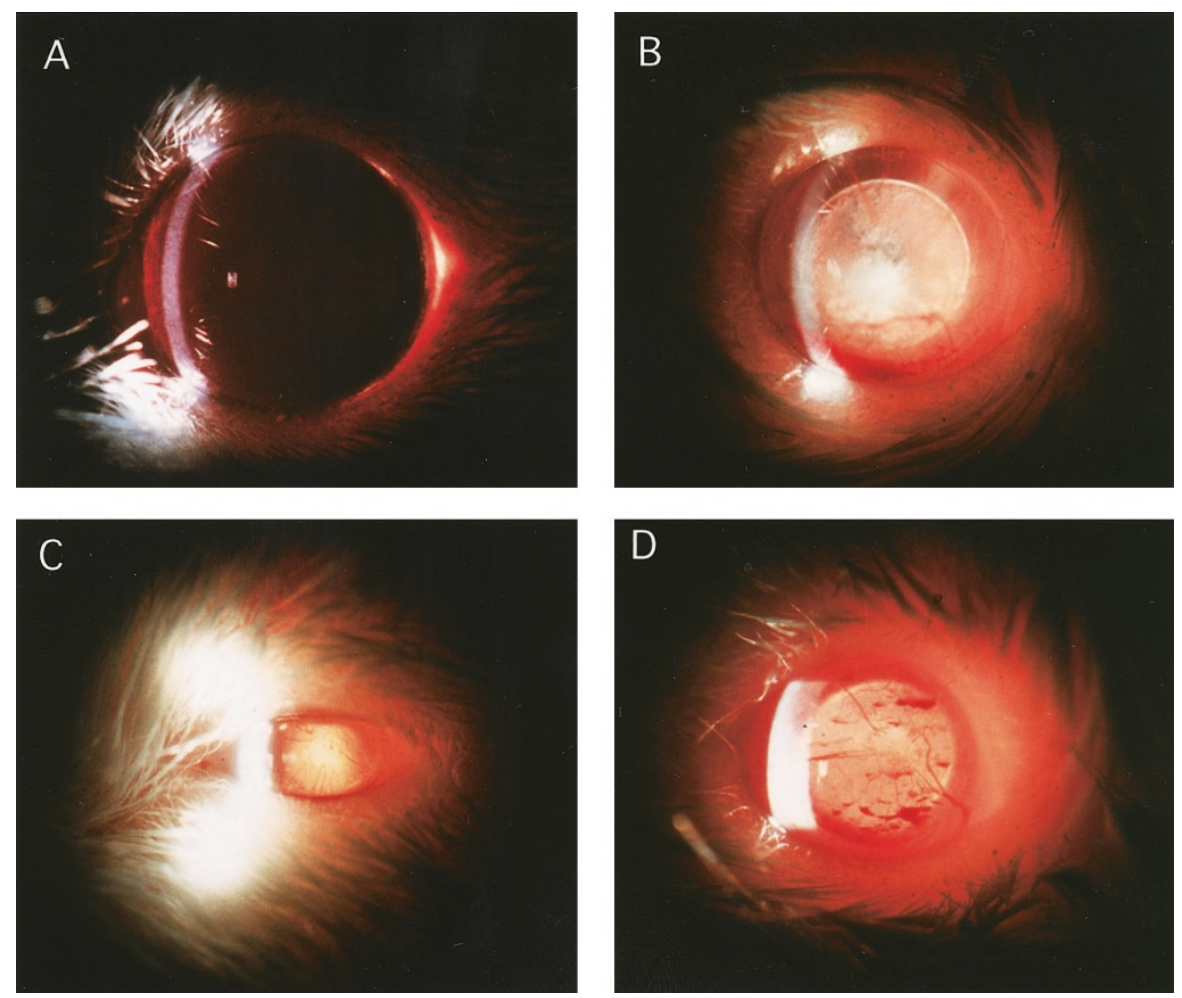

Figure 4.

Ocular phenotypes of C3 transgenic mice. The eyes of 6-week-old mice were dilated with $1 \%$ tropicamide and $2.5 \%$ phenylephrine hydrochloride, and pictures were recorded with a slitlamp biomicroscope. As compared with wild-type control (A), the transgenic animals developed severe lenticular opacity or cataract (B-D), microphthalmia (C), and intraocular hemorrhage (D). Animals from both lines 0D04-11 and 0D04-21 exhibited these phenotypes, whereas line 0D04-16 animals showed no cataract, microphalmia, or ocular hemorrhage (data not shown). Photographs shown here are from line 0D04-21 mice.

cells in C3 lenses were swollen and shorter than the wild type (Fig. 5b, panel E). C3 mice at postnatal day 12 (Fig. 5a, panel G) exhibited a severe degeneration of lens structure, with accumulation of large vacuoles and abnormal fiber cell organization at the equatorial and posterior regions close to the capsule. Frequently there was rupture of the posterior capsule with extensive leakage of lens material into the vitreous as seen in Figure 5a, panels $\mathrm{G}$ and $\mathrm{H}$. In most of the $\mathrm{C} 3$ lenses in both lines ODO4-11 and ODO4-21, the effect of C3 was greatest at the equatorial and posterior subcapsular region with severe vacuolization and abnormal fiber cell organization. The epithelium in general was found to be quite normal even in adult lenses. In 12-day-old lenses, fiber cell disorganization was associated with accumulation of nuclei in the posterior and center of the lens (Fig. 5b, panel F). Six-week-old lenses were grossly degenerated with ruptured capsule and severe vacuolization throughout the lens. Figure $5 \mathrm{~b}$ illustrates the histologic changes in C3 transgenic mice at higher magnification. Interestingly, the anterior capsule of C3 mice (ODO4-11) was noticeably much thicker $(13.1 \pm 0.807 \mu \mathrm{m}, n=16)$ than in the wild type (5.5 $\pm 0.208 \mu \mathrm{m}, n=16)$, and this abnormality is especially prominent in older lenses (Fig. 6). Although samples for line ODO4-21 were limited, three independent samples obtained from mice older than 6 months consistently showed thicker capsules than corresponding littermate controls similar to line ODO4-11 mice.
In both lines 0D04-11 and 0D04-21 mice, histologic abnormalities were also observed in ocular tissues other than the lens. In many specimens, there appeared to be synechiae, in which the iris was fused to either the cornea or lens. This may explain the failure of the iris to dilate well in response to chemical agents. Similarly, in many C3-positive mice (both lines), the retina was attached to the posterior of the lens and found to be swollen and undifferentiated as compared with controls as shown in Figure 5a, panel H. Furthermore, some of the eyes from C3 mice (both lines) showed severe hemorrhage with accumulation of erythrocytes in the vitreous and/or the aqueous chambers of the eye (Fig. 5a, panel F, indicated with arrows). Electron microscopic analysis was undertaken to determine the source of the bleeding. Several obvious changes in the vessel walls within the posterior hyaloid membrane were found (Figs. 7 and 8). In vessels of eyes in which hemorrhage was less severe and only moderate numbers of erythrocytes and macrophages were present in the vitreous body, the vascular wall adjacent to the lens capsule was markedly thinned (Fig. 7). These vessels often contained thrombocytes. In eyes with severe hemorrhage, the whole vitreous cavity was filled with erythrocytes (Fig. 8). Between the erythrocytes, many macrophages were observed that often contained phagocytosed erythrocytes (Fig. 8, A and B). By electron microscopy, separation of endothelial cells and larger openings of the vascular wall were frequently observed in the 
a
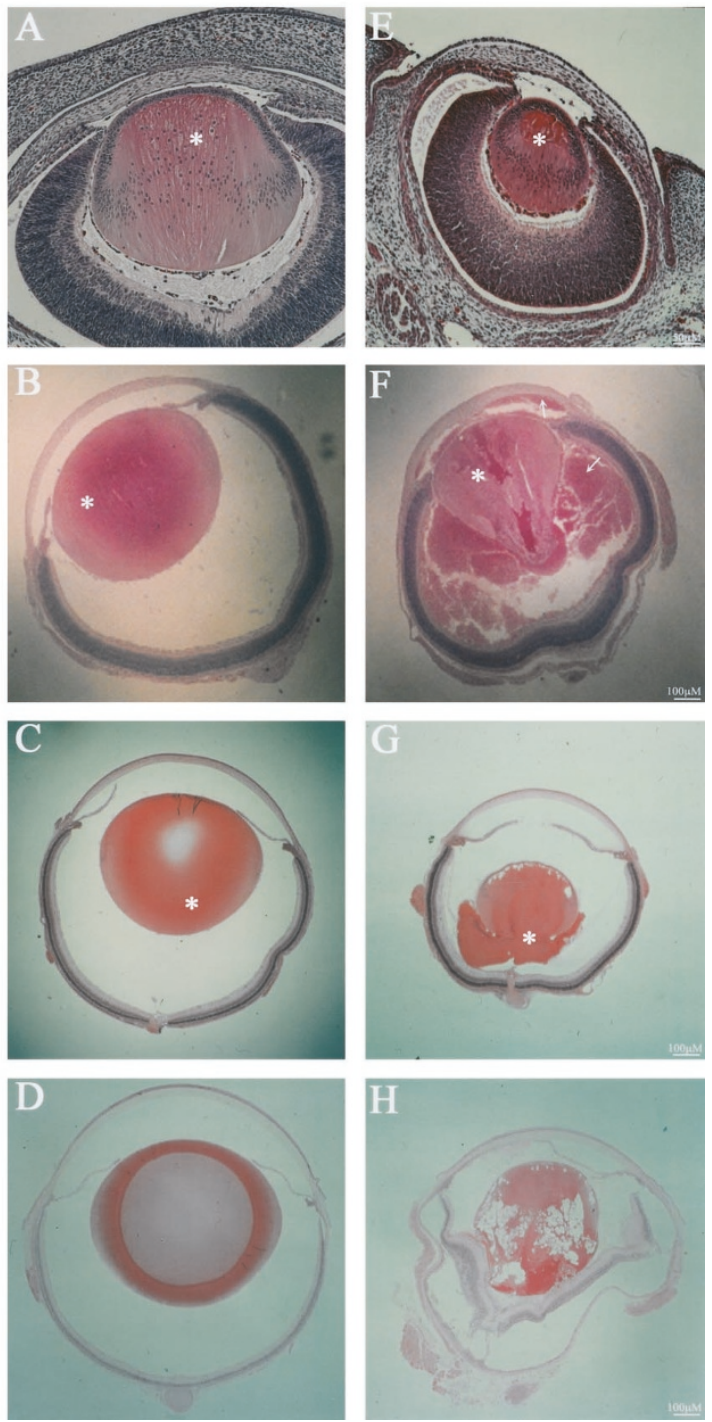

\section{Figure 5a.}

a, Histologic changes in $\mathrm{C} 3$ transgenic mouse eyes. Whole eyes from wild-type littermates (A to D) and age-matched transgenic mice, embryonic (E15.5) (E), 4 day $(F), 12$ day $(G)$, and 6 week $(H)$ were fixed in glutaraldehyde as described in "Materials and Methods." Sections were stained with hematoxylin and eosin. As the figure demonstrates, progressive degenerative histologic changes were observed in the C3 eyes beginning during embryogenesis. In eyes from neonatal and juvenile transgenic animals, such changes included ruptured lens capsule, severe vacuole accumulation, impaired fiber elongation and differentiation, and presence of nuclei in differentiated lens fibers. Arrows in panel $\mathrm{F}$ indicate hemorrhage in C3 transgenic eye. b. Histologic changes in C3 mouse lenses at higher magnification. Sections from the regions indicated with stars in Figure 5a were viewed at higher magnification to depict the abnormal changes in fiber cell organization, elongation, and differentiation. $A$ to $C$ and $D$ to $F$ are from control and transgenic samples, respectively, from E15.5, day 4 , and day 12 mice. Arrows in panel $\mathrm{F}$ depict the presence of nuclei in fiber cells, indicating abnormal terminal differentiation. Data shown here are from line 0D04-21 mice, and similar changes were observed in line 0D04-11 mice (data not shown).

vessels of the posterior hyaloid membrane (Fig. 8, C and $\mathrm{D}$ ). These openings were not plugged by thrombocytes but contained fine fibrillar material. Erythrocytes that appeared to leak through the openings were phagocytosed by macrophages that were regularly observed in the immediate vicinity (Fig. 8, C and D). b
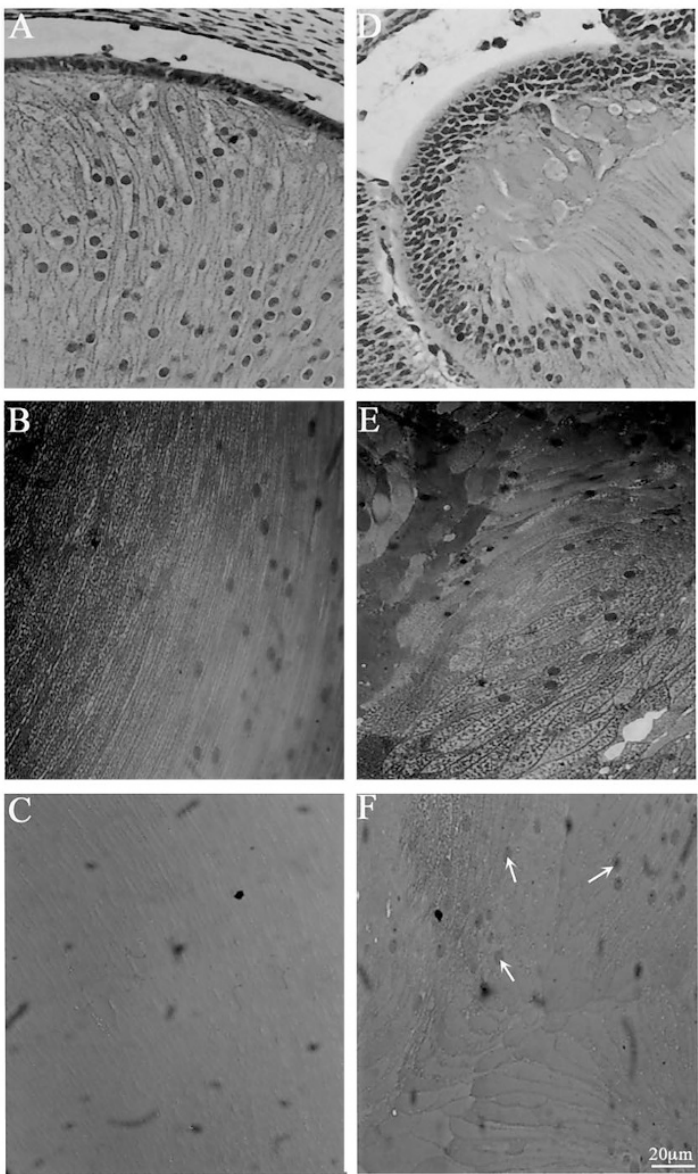

Figure 5b.

\section{Discussion}

Data presented in this study demonstrate that the selective inhibition of Rho GTPase in the developing lens impairs lens growth and integrity, suggesting the importance of Rho GTPase signaling in maintaining lens transparency and growth.

Lens fiber cell differentiation and elongation appear grossly impaired in C3 transgenic mice (Fig. 5, a and b). The Rho family of small GTPases (Rho, Rac, and CDC42) has been implicated in regulating various aspects of cytoskeletal reorganization. The three isoforms of Rho (RhoA, RhoB, and RhoC) regulate organization of the actin cytoskeleton through multiple mechanisms, including modulation of activities of phosphatidyl inositol phospholipid kinases, myosin light chain phosphatase, LIM kinase, CP1-17, and cofilin phosphorylation (Hall, 1998; Kaibuchi et al, 1999; Uehata et al, 1997). Rho GTPases mediate their effects through serine/threonine kinases such as the Rho kinases (Aelst and D'Souza-Schorey, 1997; Hall, 1998; Kaibuchi et al, 1999; Uehata et al, 1997). We have demonstrated endogenous expression of both Rho GTPase (Maddala et al, 2001a; Rao et al, 1997a) and regulatory factors for Rho GTPase, including the Rho GAPs, Rho GEFs, Rho GDI, and Rho kinases in lens (our unpublished data). The activity of Rho GTP- 

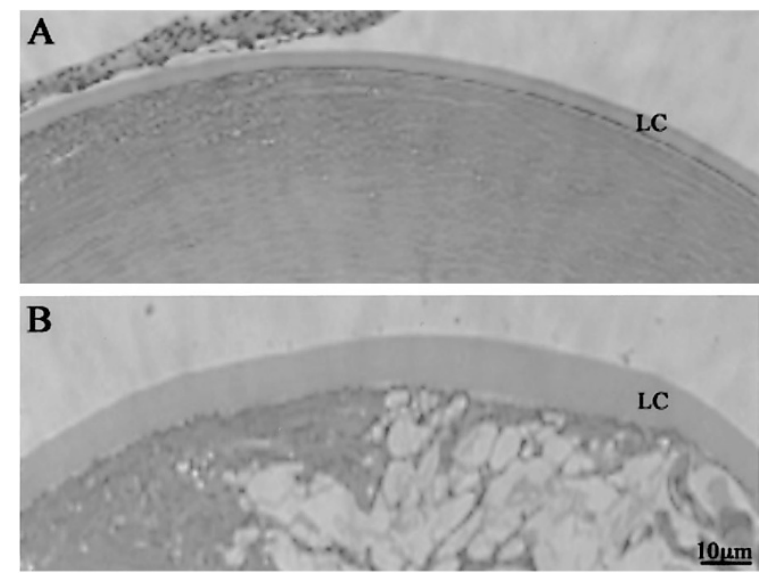

Figure 6.

Changes in lens capsule thickness in C3 transgenic mice. Anterior capsule of line 0D04-11 C3 transgenic lenses (B) was thickened dramatically in the older animals (6 months and older) as compared with littermate controls (A). Older 0D04-21 mice were not available to examine these changes. $L C=$ Lens capsule.

ases in the transgenic mice lenses seemed to be decreased by $50 \%$ or more based on the in vitro ADP-ribosylation of lens homogenates mediated by C3-exoenzyme (Fig. 3B). Most of this inactivation was likely associated with lens fiber Rho GTPases because the $\alpha \mathrm{A}$-crystallin promoter used to drive the C3 expression is specific to lens fibers (Overbeek et al, 1985; Wawrousek et al, 1990). The generally normal appearance of the lens epithelium in the C3 mice suggest lack of expression of the transgene in these cells. The $\alpha \mathrm{A}$-crystallin promoter used in this study is known to be expressed no earlier than E11.5 (Overbeek et al, 1985), and therefore, in early developmental stages lens fiber cell elongation would not be expected to affect C3 transgenic mice.

In contrast to the inherent limitations of cell culture systems, the C3 transgenic mouse developed in this study represents a tractable in vivo model for studying the contribution of Rho GTPase signaling pathways participating in lens development and growth. Furthermore, the lens system itself provides distinct advantages in terms of the simplicity of cellular composition and its avascular nature. C3-exoenzyme has previously been expressed in the thymus using a tissuespecific promoter demonstrating the critical role of Rho GTPase function in the development of thymus (Henning et al, 1997). Expression of C3 in the lens suggests a definite role for Rho GTPase in the regulation of growth, differentiation, and functional integrity of this organ. Disorganization and vacuolization of fiber cells and the presence of nuclei in differentiated fibers suggests impaired fiber cell differentiation. Consistent with our hypothesis, lens-specific expression of the $\mathrm{dbl}$ oncogene product, which can function as an exchange factor for Rho GTPases in vitro, has been previously shown to induce cataract formation and impair lens fiber cell elongation and differentiation (Eva et al, 1991). Furthermore, another Rho GTPase regulatory protein, Rho GAP6, has been localized to a chromosomal region linked to microphthalmia (Schaefer et al, 1997). The preceding observations, together with the

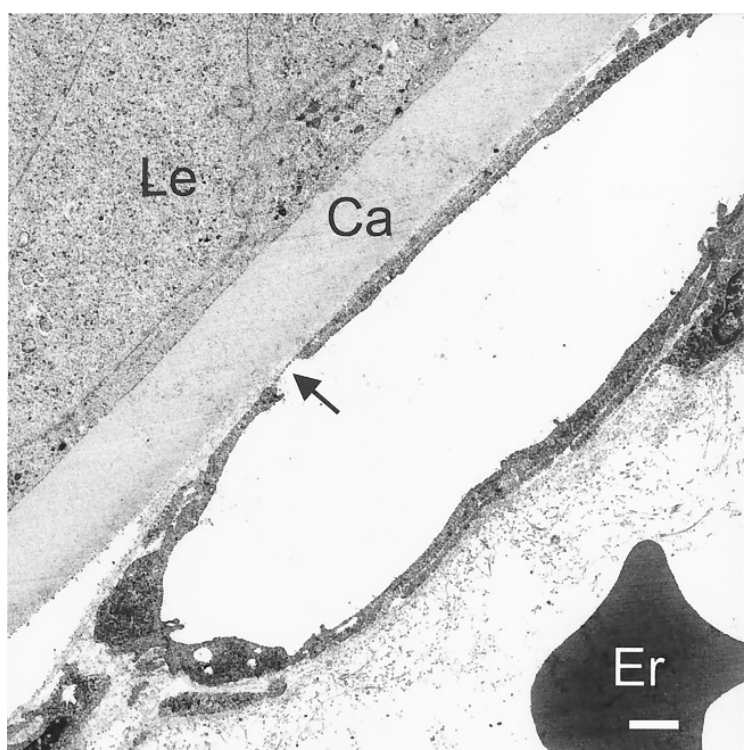

Figure 7.

Electron microscopy of a posterior hyaloid vessel in a C3 transgenic (ODO421) embryo (E17.5) with moderate intraocular hemorrhage (bar is $1.26 \mu \mathrm{m}$ ). The vascular wall adjacent to the lens capsule $(\mathrm{Ca})$ is markedly thinned in a distinctly localized area (large arrow). Le = Lens; Er = Erythrocyte.

data presented in this study, suggest an important role for Rho GTPases in regulation of developmental processes in the lens. The possibility that perturbation of the actin cytoskeleton is a factor in these C3-exoenzymemediated effects is under investigation.

Although the posterior lens capsule is ruptured in many of the $\mathrm{C} 3$ mice (Fig. 5a, G and H), the anterior capsule is markedly thickened, particularly in older animals (Fig. 6); whether this is caused by changes in basal lamina proteins of the capsule is not known. Interestingly, transgenic mice expressing diphtheria toxin in the lens have also been reported to exhibit ruptured capsules (Breitman et al, 1990; Harrington et al, 1991) as do mice deficient in SPARC/osteonectin. In the latter situation, there were no changes in the major basal lamina proteins laminin, collagen, perlecan, or entactin (Gilmour et al, 1998).

Microphthalmia is commonly manifested in transgenic mouse models harboring defects of growth factor biology or cell cycle regulation (Fromm et al, 1994; Lovicu and Overbeek, 1998). The C3 mice frequently had unilateral or bilateral microphthalmia; the reasons for this heterogeneity are not clear at present. A similar heterogeneity was observed in transgenic mice expressing diphtheria toxin in the lens, and the microphthalmia was attributed to defective lens development, suggesting that normal lens development is critical for normal development of the entire eye (Beebe and Coats, 2000; Breitman et al, 1990; Harrington et al, 1991). It is unclear whether the extralenticular defects seen in C3 mice, including synechiae and hemorrhage, are direct effects of Rho GTPase inactivation in these tissues because of C3 leakage from the lens or are secondary to other effects on the lens. Because C3 expression was not detected in ocular tissues except for the lens, leakage from the 


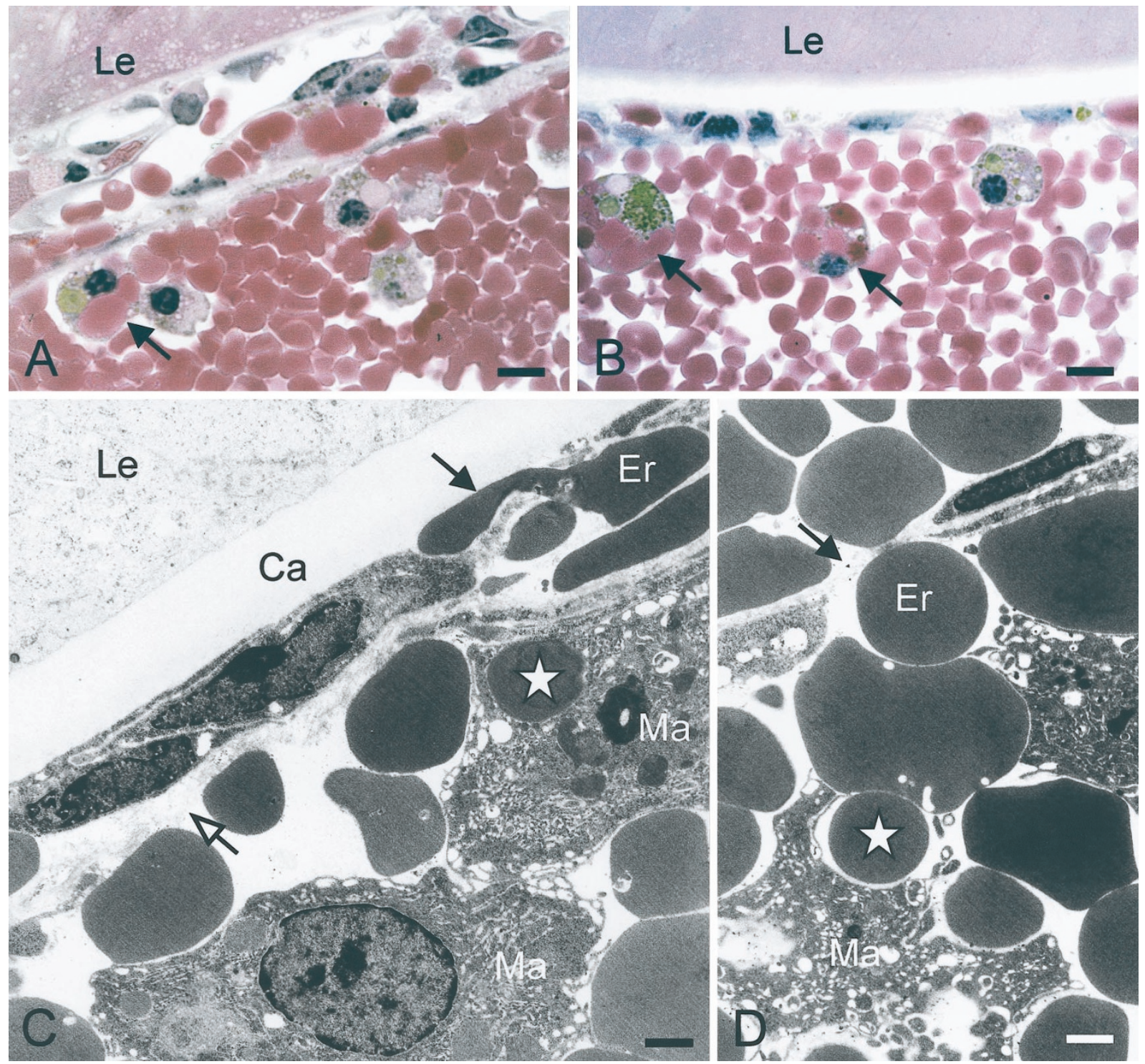

\section{Figure 8.}

Light ( $A$ and $B$ ) and electron microscopy ( $C$ and $D$ ) of posterior hyaloid vessels in a C3 transgenic (OD04-21) embryo (E15.5) with severe ocular hemorrhage. A and $B$, bar is $8.4 \mu \mathrm{m}$. In eyes with severe hemorrhage, the whole vitreous cavity is filled with erythrocytes. Between the erythrocytes, many macrophages are observed that contain phagocytosed erythrocytes (arrows). C and D, The endothelial cells of the vessels are separated from each other and form large openings towards both lens capsule and the vitreous cavity (arrows) (bar is $1.4 \mu \mathrm{m}$ ). $\mathrm{Le}=\mathrm{Lens} ; \mathrm{Ca}=$ Capsule; $\mathrm{Er}=$ Erythrocytes; $\mathrm{Ma}=$ Macrophage. Asterisk indicates phagocytosed erythrocytes.

lens is probably responsible for these changes. Alternatively, defective lens development by itself could be responsible for abnormalities elsewhere in the eye (Beebe and Coats, 2000; Thut et al, 2001). Secretion of C3-exoenzyme from the lens is unlikely because the C3 transgene used in this study does not contain the signal peptide required for secretion. Intriguingly, the hemorrhagic phenotype found in C3 mice seems to be unique and has not been reported in other animal models of cataract (Fig. 5a, panel F, and Fig. 8). Rho GTPase has been implicated in endothelial cell migration and contraction and thereby may regulate gap formation between cell junctions (Aepfelbacher et al, 1997; Essler et al, 1998; Vouret-Craviari et al, 1998). Furthermore, Rho GTPase signaling has also been shown to interact with $\mathrm{G} \alpha 12$ and $\mathrm{G} \alpha 13$ signaling, thereby regulating vascular endothelial cell integrity and wound repair (Offermanns et al, 1997). It is possible that the cell-cell junctions and the actin-myosin system that maintain the integrity of the endothelial barrier of hyaloid vessels are impaired by C3mediated reactions, resulting in movement of erythrocytes into extracellular spaces (Figs. 7 and 8).

In conclusion, the present study demonstrates that Rho GTPase is important in regulating lens growth and development and in maintaining lens transparency. We speculate that the histologic changes associated with lens fiber and capsule integrity might be directly related to Rho GTPase-regulated actin cytoskeletal organization and focal adhesions. Furthermore, this C3 transgenic mouse should serve as an important model for investigating the effects of Rho GTPase 
signaling in vivo on lens fiber cell cytoskeletal reorganization and terminal differentiation. Such studies are currently in progress.

\section{Materials and Methods}

\section{Generation of C3-Exoenzyme Transgenic Mice}

A C3-exoenzyme (Botulinum C3 ADP-ribosyltransferase) expression vector (pGEX T2-C3) was kindly provided by Dr. Larry Feig from Tufts University Medical School (Dillon and Feig, 1995). The cDNA insert (truncated 616 bp) encoding C3-exoenzyme was released from this parental expression plasmid using Sau3Al and Nlall restriction enzymes. A synthetic oligonucleotide containing the remainder of the missing coding sequence of mature $\mathrm{C} 3$ (36 bp), plus a eukaryotic translation initiation sequence CCACCATG, was added (Fig. 1B). This sequence, encoding mature C3 (652 bp without the signal peptide sequence), was ligated into the polylinker (Clal and Hindlll sites) of CpV2, a plasmid vector carrying a modified version of the murine $\alpha \mathrm{A}$-crystallin promoter separated by a polylinker from the SV40 small $t$ antigen intron and polyadenylation sequence. After confirming the orientation and sequence of the C3 CDNA insert by dideoxy sequencing, the transgene DNA fragment was released by Notl digestion and purified by agarose gel electrophoresis. Microinjection of the transgene into single-celled FVB/N mouse embryos and subsequent generation of transgenic mice was performed as described earlier (Wawrousek et al, 1990). Transgenic mice were identified by PCR analysis of genomic DNA isolated from tail biopsy specimens using the following primers: CCGAGCTGAGCATAGACATT and TAACTCCTTATTTTTGTCTTAGC.

\section{RT-PCR}

Total RNA was extracted from a single pair or from four to six pooled lenses using the Qiagen RNeasy mini kit (QIAGEN, Valencia, California). DNase-1treated total RNA was reverse transcribed using an RT-PCR kit (Clontech, Palo Alto, California). cDNA generated from the $\mathrm{RT}$ reaction was used as template to amplify C3 transgene, RhoA, and RhoB mRNAs using the following $5^{\prime}$-specific and $3^{\prime}$-specific primers of $\mathrm{C} 3$, RhoA, RhoB, and GAPDH, respectively: C3.1 (AAGCAAAAGCTTGGGGTAATGC) and C3.2 (TTTAGCCTITTCAAAAGCCGTITTAT); RhoA (TGAAAACTATGTGGCGGATATCG and TCTGCTTCTTCAGGTTTAACCGG); RhoB (AGGACTACGATCGTTTACGGCC and CAGCCATTCTGGGATCCGTAG); GAPDH (ACCACAGTCCATGCCATCAC and TCCACCACCCTGTTGCTGTA).

\section{SDS-PAGE and Western Blot Analysis}

For SDS-PAGE analysis and immunoblotting, suitable aliquots of lens homogenates were dissolved in $65 \mathrm{~mm}$ Tris buffer, $\mathrm{pH} 7.4$, containing 2\% SDS and 2\% $\beta$-mercaptoethanol and separated on $12.5 \%$ acrylamide gels according to the method of Laemmli (1970), using a Hoefer Mighty Small apparatus. Proteins were electrophoretically transferred to nitrocel- lulose and immunoblotted for C3-exoenzyme using a monoclonal antibody and ECL peroxidase system (Amersham, Arlington Heights, Illinois).

\section{In Vitro ADP-Ribosylation}

C3-exoenzyme-catalyzed ADP-ribosylation of Rho GTPase was performed as described by Rao et al (1997b). Lens samples were homogenized in ice-cold $5 \mathrm{~mm}$ Tris buffer, $\mathrm{pH} 7.4$, containing $5 \mathrm{~mm} \mathrm{MgCl}_{2}, 1 \mathrm{~mm}$ EDTA, $1 \mathrm{~mm}$ dithiothreitol, $0.5 \mathrm{~mm}$ PMSF, $1 \mu \mathrm{g} / \mathrm{ml}$ aprotinin, and $1 \mu \mathrm{g} / \mathrm{ml}$ leupeptin. The $700 \times g$ supernatants were subjected to the ADP-ribosylation assay. Briefly, the assay was performed in a final volume of $0.1 \mathrm{ml}$ containing $100 \mathrm{~mm}$ Tris, $\mathrm{pH} 8.0,2.5 \mathrm{~mm} \mathrm{MgCl}_{2}$, $1.0 \mathrm{~mm}$ EDTA, $10 \mathrm{~mm}$ thymidine, $10 \mathrm{~mm}$ dithiothreitol, 1 $\mathrm{mm}$ ATP, $0.1 \mathrm{~mm}$ GTP, $1 \mu \mathrm{Ci}\left[{ }^{32} \mathrm{P}\right] \mathrm{NAD}(1000 \mathrm{Ci} /$ mmol-1; DuPont-New England Nuclear, Boston, Massachusetts), and $50 \mu \mathrm{g}$ of lens protein. The assay was initiated by addition of $1 \mu \mathrm{g}$ of C3-exoenzyme (Calbiochem, San Diego, California); after a 60-minute incubation at $37^{\circ} \mathrm{C}$, it was stopped by addition of 11 $\mu \mathrm{l}$ of ice-cold TCA $(70 \% \mathrm{v} / \mathrm{v})$. TCA precipitates were dissolved in Laemmli sample buffer and then subjected to SDS-PAGE (12.5\%). ADP-ribosylated proteins were detected by autoradiography.

\section{Histologic Analysis}

Tissues for histologic analysis, which included heads of embryos (E15.5) or whole eyes of postnatal animals, were immersed in $2.5 \%$ glutaraldehyde in $50 \mathrm{~mm}$ cacodylate buffer $(\mathrm{pH} 7.2)$ containing $4 \%$ sucrose and $2 \mathrm{mM} \mathrm{CaCl}_{2}$ for 2 hours, then transferred to $10 \%$ buffered formalin for another 48 hours or more. They were then embedded in methacrylate, cut into 1- to $2-\mu \mathrm{m}$ sections, and stained with hematoxylin and eosin using conventional methods.

\section{Electron Microscopy}

Heads from E15.5 and E17.5 transgenic embryos with obvious ocular hemorrhage and from wild-type littermates were immersed overnight in $2.5 \%$ glutaraldehyde in $50 \mathrm{~mm}$ cacodylate buffer $(\mathrm{pH} 7.2)$ containing $4 \%$ sucrose and $2 \mathrm{~mm} \mathrm{CaCl}_{2}$. After fixation, the eyes were washed for at least 24 hours in cacodylate buffer, postfixed with $2 \%$ osmium tetroxide, and embedded in Epon (Roth, Karlsruhe, Germany). Ultrathin sections were cut on a microtome, contrasted with lead citrate and uranyl acetate, and viewed with a Zeiss EM 902 electron microscope (Zeiss, Oberkochen, Germany).

\section{Acknowledgements}

The authors are very thankful to Drs. Larry Feig for providing pGEX2T-C3 plasmid, Shunji Kozaki for antiC3-exoenzyme monoclonal antibody, and A. Cvekl and Joram Piatigorsky for the $\alpha \mathrm{A}$-crystallin promoter. We thank Dr. Gordon Klintworth for his suggestions and Dr. Rupalatha Maddala for technical help. We are also grateful to Ms. Anke Fischer and Mr. Marco GoBwein for technical help with electron microscopy. 


\section{References}

Aelst IV and D'Souza-Schorey CD (1997). Rho GTPases and signaling network. Genes Dev 11:2295-2322.

Aepfelbacher M, Essler M, Huber E, Sugai M, and Weber PC (1997). Bacterial toxins block endothelial wound repair: Evidence that Rho GTPases control cytoskeletal rearrangements in migrating endothelial cells. Arterioscler Thromb Vasc Biol 17:1623-1629.

Beebe DC and Coats J M (2000). The lens organizes the anterior segment: Specification of neural crest cell differentiation in the avian eye. Dev Biol 220:424-431.

Breitman ML, Rombola H, Maxwell IH, Klintworth GK, and Berntein A (1990). Genetic ablation in transgenic mice with an attenuated diphtheria toxin a gene. Mol Cell Biol 10:474479.

Dillon ST and Feig LA (1995). Purification and assay of recombinant C3 transferase. Methods Enzymol 256:174-183.

Essler M, Amano M, Kruse HJ, Kaibuchi K, Weber PC, and Aepfelbacher M (1998). Thrombin inactivates myosin light chain phosphatase via Rho and its target Rho kinase in human endothelial cells. J Biol Chem 273:21867-21874.

Eva A, Graziani G, Zannini M, Merin LM, Khillan JS, and Overbeek PA (1991). Dominant dysplasia of the lens in transgenic mice expressing the $\mathrm{dbl}$ oncogene. New Biol 3:158-168.

Fromm L, Shawlot W, Gunning K, Butel JS, and Overbeek PA (1994). The retinoblastoma protein-binding region of simian virus 40 large $T$ antigen alters cell cycle regulation in lenses of transgenic mice. Mol Cell Biol 14:6743-6754.

Hall A (1998). Rho GTPases and the actin cytoskeleton. Science 279:509-514.

Harrington L, Klintworth GK, Sector TE, and Breitman ML (1991). Developmental analysis of ocular morphogenesis in alphaA-crystallin/diphtheria toxin transgenic mice undergoing ablation of the lens. Dev Biol 148:508-516.

Henning W, Galandrini R, Hall A, and Cantrell DA (1997). The GTPase Rho has a critical regulatory role in thymus development. EMBO J 16:2397-2407.

Gilmour DT, Lyon GJ, Carlton MBL, Sanes JR, Cunningham JM, Anderson JR, Hogan BL, Evans MJ, and Colledge WH (1998). Mice deficient for the secreted glycoprotein SPARC/ osteonectin/BM40 develop normally but show severe ageonset cataract formation and disruption of the lens. EMBO $\mathrm{J}$ $17: 1860-1870$.

Kaibuchi K, Kuroda S, and Amano M (1999). Regulation of the cytoskeleton and cell adhesion by the Rho family GTPases in mammalian cells. Ann Rev Biochem 68:459-486.

Laemmli UK (1970). Cleavage of structural proteins during the assembly of the head of bacteriophage T4. Nature 227:680-685.

Lovicu FJ and Overbeek PA (1998). Overlapping effects of different members of the FGF family on lens fiber differentiation in transgenic mice. Development 125:3365-3377.

Maddala RL, Peng YW, and Rao PV (2001a). Selective expression of the small GTPase RhoB in the early developing mouse lens. Dev Dynamics 222:534-537.

Maddala RL, Reddy VN, and Rao PV (2001b). Lovastatininduced cytoskeletal reorganization in lens epithelial cells: Role of Rho GTPases. Invest Ophthalmol Vis Sci 42:2610-2615.
McAvoy JW (1978). Cell division, cell elongation, and the coordination of crystallin gene expression during lens morphogenesis in the rat. J Embryol Exp Morphol 45:271-281.

Offermanns S, Mancino V, Revel JP, and Simon MI (1997). Vascular system defects and impaired cell chemokinesis as a result of Galpha13 deficiency. Science 275:533-536.

Overbeek PA, Chepelinsky AB, Khillan JS, Piatigorsky J, and Westphal H (1985). Lens-specific expression and developmental regulation of the bacterial chloramphenicol acetyltransferase gene driven by the murine $\alpha \mathrm{A}$-crystallin promoter in transgenic mice. Proc Natl Acad Sci USA 82:7815-7819.

Piatigorsky J (1981). Lens differentiation in vertebrates: A review of cellular and molecular features. Differentiation 19:134-153.

Rao PV, Robison WG, Bettelheim F, Lin LR, Reddy VN, and Zigler JS (1997a). Role of small GTP-binding proteins in lovastatin-induced cataract. Invest Ophthalmol Vis Sci 38: 2313-2321.

Rao PV, Zigler JS, and Garland D (1997b). Analysis of small GTP-binding proteins of the lens by GTP overlay assay reveals the presence of unique GTP-binding proteins associated with fiber cells. Exp Eye Res 64:219-227.

Robinson ML, Overbeek PA, Verran DJ, Grizzle WE, Stockard CR, Friesel R, Maciag T, and Thompson JA (1995). Extracellular FGF-1 acts as a lens differentiation factor in transgenic mice. Development 121:505-514.

Rubin EJ, Gill DM, Boquet P, Popoff MR (1988). Functional modification of a 21-kilodalton $G$ protein when ADPribosylated by exoenzyme $\mathrm{C} 3$ of Clostridium botulinum. Mol Cell Biol 8:418-426.

Schaefer L, Prakash S, and Zoghbi HY (1997). Cloning and characterization of a novel rho-type GTPase-activating protein gene (ARHGAP6) from the critical region for microphthalmia with linear skin defects. Genomics 46:268-277.

Stolen CM, Jackson MW, and Griep AE (1997). Overexpression of FGF-2 modulates fiber cell differentiation and survival in the mouse lens. Development 124:4009-4017.

Thut CT, Rountree RB, Hwa M, and Kingsley DM (2001). A large-scale in situ screen provides molecular evidence for the induction of eye anterior segment structures by the developing lens. Dev Biol 231:63-76.

Uehata M, Ishizaki T, Satoh H, Ono T, Kawahara T, Morishita $\mathrm{T}$, Tamakawa $\mathrm{H}$, Yamagami $\mathrm{K}$, Inui J, Maekawa $\mathrm{M}$, and Narumiya S (1997). Calcium sensitization of smooth muscle mediated by a Rho-associated protein kinase in hypertension. Nature 389:990-994.

Vouret-Craviari V, Boquet P, Pouyssegur J, and Van Obberghen-Schilling E (1998). Regulation of the actin cytoskeleton by thrombin in human endothelial cells: Role of Rho proteins in endothelial barrier function. Mol Biol Cell 9:2639-2653.

Wawrousek EF, Chepelinsky AB, McDermott JB, Piatigorsky $J(1990)$. Regulation of the murine alphaA-crystallin promoter in transgenic mice. Dev Biol 137:68-76.

Yamamoto M, Marui N, Sakai T, Morii N, Kozaki S, Ikai K, Imamura S, and Narumiya S (1993). ADP-ribosylation of the rhoA gene product by botulinum $\mathrm{C} 3$ exoenzyme causes Swiss 3 T3 cells to accumulate in the G1 phase of the cell cycle. Oncogene 8:1449-1455. 